\title{
NAFLD: a multisystem disease requiring a
}

\section{multidisciplinary and holistic approach}

\author{
Giovanni Targher ${ }^{1 *}$, Herbert Tilg ${ }^{2 *}$, Christopher D Byrne ${ }^{3,4 *}$
}

${ }^{*}$ All three authors contributed equally to this manuscript

${ }^{1}$ Section of Endocrinology, Diabetes and Metabolism, Department of Medicine, University and Azienda Ospedaliera Universitaria Integrata of Verona, Verona, Italy

2Department of Internal Medicine I, Gastroenterology, Hepatology, Endocrinology \& Metabolism, Medical University Innsbruck, Innsbruck, Austria

${ }^{3}$ Nutrition and Metabolism, Faculty of Medicine, University of Southampton, UK

${ }^{4}$ Southampton National Institute for Health Research Biomedical Research Centre, University Hospital Southampton, Southampton General Hospital, Tremona Road, Southampton, UK

Word count: 149 abstract; 5625 text (excluding title page, abstract, references, tables and figure legends). Tables=1, Figures=4, References $=94$

Address for correspondence:

Prof. Giovanni Targher, MD

Section of Endocrinology, Diabetes and Metabolism

Department of Medicine

University and Azienda Ospedaliera Universitaria Integrata

Piazzale A. Stefani, 1

37126 Verona, Italy

Phone: +39-045-8123110

E-mail: giovanni.targher@univr.it 


\section{Summary}

Nonalcoholic fatty liver disease (NAFLD) has reached epidemic proportions worldwide. This narrative Review provides an overview of the current literature supporting the notion that NAFLD is a multi-system disease. Convincing evidence now supports a strong association between NAFLD and the risk of developing multiple extra-hepatic complications, such as type 2 diabetes mellitus (T2DM), cardiovascular disease (CVD, i.e., the predominant cause of mortality in people with NAFLD), chronic kidney disease (CKD), and certain types of extrahepatic malignancies. The magnitude of this risk parallels the severity of NAFLD (especially the stage of liver fibrosis). There are likely multiple underlying mechanisms by which NAFLD may increase the risk of CVD, T2DM and other extra-hepatic complications. Combating the growing burden of NAFLD will require setting up a multidisciplinary working group and framework to progress and embrace novel, collaborative ways of working to deliver a holistic, person-centred care and management of individuals with NAFLD. 


\section{Introduction}

Nonalcoholic fatty liver disease (NAFLD) is a public health problem worldwide that affects up to around $30 \%$ of the adult population and causes considerable liver-related and extrahepatic morbidity and mortality ${ }^{1-3}$. It is known that NAFLD is associated with an increased risk of all-cause mortality and the leading cause of mortality in this patient population is cardiovascular disease (CVD), followed by extra-hepatic cancers and liver-related complications $^{1-3}$.

Over the last 5-6 years, it is becoming increasingly evident that NAFLD is a "multi-system" disease 4 that not only increases the risk of liver-related complications (nonalcoholic steatohepatitis [NASH], cirrhosis or hepatocellular carcinoma [HCC]), but also affects the risk of developing CVD and other extra-hepatic diseases, such as type 2 diabetes mellitus (T2DM), chronic kidney disease (CKD), or certain extra-hepatic cancers (e.g., colorectal cancer $)^{1,3,5}$. To further emphasize the systemic nature of NAFLD and the close links between this common metabolic liver disease and other cardiometabolic diseases, an international panel of experts has recently proposed to change the terminology from NAFLD to "metabolic dysfunction-associated fatty liver disease" (MAFLD) ${ }^{6-11}$. Although the proposed change of nomenclature from NAFLD to MAFLD is still under discussion, the rationale of using this new terminology largely stems from the fact that there is a pathophysiological link between this liver disease and the presence of underlying metabolic abnormalities (namely overweight/obesity, T2DM, insulin resistance or other metabolic risk factors). Additionally, there is also evidence suggesting that the MAFLD definition is more accurate than the NAFLD definition in identifying those individuals with hepatic steatosis, who are at high risk of disease progression or have a higher risk of CVD and CKD ${ }^{12-15}$.

To date, there remains substantial underappreciation of the importance of NAFLD by primary care clinicians, other specialists and patients ${ }^{16,17}$. Thus, the main aim of this Review is to summarise new insights into NAFLD to facilitate improved health promotion, case identification, patient awareness, and health services delivery. In this Review, we also provided a perspective towards building a framework for a person-centered, multidisciplinary and holistic approach to individuals with NAFLD.

\section{Extra-hepatic complications: epidemiological evidence}

Table 1 summarizes the meta-analytic quantification of the excess of fatal and nonfatal CVD events, cardiac arrhythmias (mostly permanent atrial fibrillation), T2DM, CKD, and colorectal tumours in people with NAFLD. In this Review, we have not discussed the association 
between NAFLD and risk of other extra-hepatic diseases, such as primary hypothyroidism or polycystic ovary syndrome ${ }^{18,19}$, because the current available evidence for such associations is not sufficiently robust.

\subsection{Risk of CVD}

As shown in Table 1, a meta-analysis of 16 observational studies that included $\sim 34,000$ middle-aged individuals with a median 6.9-year follow-up showed that those with NAFLD had a $64 \%$ higher risk of developing fatal or nonfatal CVD events than those without NAFLD ${ }^{20}$. In addition, the risk of incident CVD events appeared to increase further with greater severity of NAFLD (random-effects odds ratio $2.58 ; 95 \% \mathrm{Cl} 1.78-3.75$ ), and remained significant in those studies where analysis was adjusted for conventional CVD risk factors ${ }^{20}$. Evidence is accumulating that the risk of CVD events parallels the underlying severity of NAFLD, and the stage of liver fibrosis is the strongest histologic predictor of adverse liverrelated and CVD outcomes in NAFLD ${ }^{21,22}$. Using a competing risk analysis, Henson et al. showed that advanced liver fibrosis was associated with greater risk of incident CVD events during a median of 5 years in a cohort of $\sim 300$ United States individuals with biopsyconfirmed NAFLD, and this risk remained significant even after adjusting for relevant covariates, including CVD risk scores ${ }^{23}$. Some cohort studies also reported that there was an association between NAFLD and the risk of progression of coronary or carotid atherosclerosis, and most importantly, that improvement or resolution of NAFLD (on ultrasonography) was associated with a lower risk of carotid atherosclerosis development ${ }^{24,25}$.

\subsection{Risk of arrhythmias}

The link between NAFLD and risk of arrhythmias (mostly permanent atrial fibrillation [AF]) has recently gained scientific interest ${ }^{26}$. As shown in Table 1 , in a meta-analysis of six longitudinal studies (involving $~ 615,000$ individuals with and without T2DM), Cai et al. showed that NAFLD was moderately associated with an increased risk of incident AF, irrespective of shared CVD risk factors ${ }^{27}$. Increasing evidence also suggests that the presence and severity of NAFLD (on ultrasonography) was independently associated with a higher risk for QTc interval prolongation (on resting electrocardiogram) that predisposes to increased risk of both ventricular tachyarrhythmias and sudden cardiac death ${ }^{26,28}$.

\subsection{Risk of T2DM}

As shown in Table 1, in a comprehensive meta-analysis including 33 longitudinal cohort studies with 501,022 individuals ( $30.8 \%$ with NAFLD) followed for a median of 5 years, Mantovani et al. found that individuals with NAFLD had a 2.2-fold higher risk of incident 
T2DM than those without NAFLD ${ }^{29}$. The risk of diabetes appeared to increase further with greater severity of NAFLD (mostly the severity of liver fibrosis) and, most importantly, remained statistically significant in those studies where analysis was adjusted for age, sex, family history of diabetes, adiposity measures, and other common metabolic risk factors ${ }^{29}$. It is worth noting that some observational studies also reported that T2DM incidence diminished over time following the improvement or resolution of NAFLD assessed by ultrasonography, irrespective of changes in body weight ${ }^{30,31}$.

\subsection{Risk of CKD}

Several observational studies reported an association between NAFLD and risk of CKD. As shown in Table 1, in a large and updated meta-analysis of 13 observational studies involving more than 1.2 million individuals from different countries, Mantovani et al. found that NAFLD was associated with a $\sim 1.5$-fold increased risk of incident CKD (stage $\geq 3$, defined as estimated GFR $<60 \mathrm{ml} / \mathrm{min} / 1.73 \mathrm{~m}^{2}$ ) over a median follow-up of 9.7 years, and that this longterm risk appeared to be even greater among individuals with NAFLD and advanced fibrosis $^{32}$. However, further research is needed to better elucidate the complex link between NAFLD and CKD, and to establish whether improvement of NAFLD attenuates development and progression of CKD.

\subsection{Risk of colorectal tumours and other extra-hepatic cancers}

As summarised in Table 1, in a meta-analysis of 8 cross-sectional and 3 longitudinal studies with aggregate data on $\sim 91,000$ asymptomatic adults of predominantly Asian descent undergoing screening colonoscopy, it has been reported that NAFLD was associated with a moderately increased prevalence and incidence of colorectal adenomas and cancer. These risks were independent of age, sex, smoking, body mass index and T2DM (or metabolic syndrome $)^{33}$. Further prospective studies, particularly in American and European populations, and mechanistic studies are needed to better understand the association between NAFLD and colonic carcinogenesis.

In a cohort study involving a total of 4,722 United States individuals with NAFLD and 14,441 age- and sex-matched individuals followed for a median of 8 years, Allen et al. found that NAFLD was associated with a $\sim 2$-fold higher risk of developing cancers, predominantly of the liver, gastrointestinal tract and uterus. Notably, the association with increased cancer risk was stronger in NAFLD than obesity, thereby suggesting that NAFLD might be a mediator of the obesity-cancer association ${ }^{34}$. In another community-based cohort of $\sim 54,000$ Chinese men, the investigators found that NAFLD was associated with a moderately increased risk of developing all cancers, thyroid cancer, and lung cancer. Furthermore, in men with NAFLD, 
higher serum aminotransferase levels were associated with higher risk of $\mathrm{HCC}$ and thyroid cancer. NAFLD also increased risk of colorectal and lung cancers only in smokers, and increased risk of kidney cancer in men without T2DM ${ }^{35}$. Finally, in a nationwide, matched cohort study that included 10,568 individuals with biopsy-proven NAFLD and 49,925 matched control subjects followed for a median of 14 years, Simon et al. found that all histological stages of NAFLD were associated with significantly increased overall mortality, and this risk increased progressively with worsening NAFLD histology (adjusted-hazard ratio (HR) $1.71,95 \% \mathrm{Cl} 1.64-1.79$ for simple steatosis; adjusted-HR 2.14, 95\% Cl 1.93-2.38 for non-fibrotic NASH; adjusted-HR 2.44, 95\%Cl 2.22-2.69 for non-cirrhotic fibrosis; and adjusted-HR $3.79,95 \% \mathrm{Cl} 3.34-4.30$ for cirrhosis, respectively). Notably, the excess mortality associated with NAFLD was primarily from extra-hepatic cancers, followed by cirrhosis, CVD and $\mathrm{HCC}^{36}$.

\section{Pathophysiological aspects in NAFLD}

The pathophysiology of NAFLD is highly complex involving rather diverse aspects, such as metabolic disturbances, lipotoxicity, insulin resistance, chronic inflammation, fibrosis, intestinal function and the gut microbiome ${ }^{37}$. NAFLD genetics has experienced a boost in recent years and many genome-wide association studies have demonstrated convincing associations with genes, such as patatin-like phospholipase domain-containing protein 3 (PNPLA3), transmembrane-6 superfamily member 2 (TM6SF2), and others ${ }^{38}$. NAFLD and related complications are frequently accompanied by low-grade metabolic inflammation ("metaflammation"), a hallmark of obesity-related disorders, T2DM and NAFLD ${ }^{39}$. Specifically, inflammatory mediators may contribute to both the liver phenotype and the extra-hepatic complications of NAFLD. Figure 1 schematically summarizes the putative biological mechanisms by which NAFLD may contribute to the development and progression of its major extra-hepatic complications.

\subsection{Adipokine/cytokine imbalance and chronic inflammation}

Adipose tissue inflammation is characterized by infiltration of various types of leukocytes. Therefore, it is assumed that adipose tissue contributes substantially to chronic inflammation in obesity-related disorders, such as NAFLD. This is important, as adipose tissue-derived inflammation might affect inflammation at distal sites, such as the liver or vessels.

Adipokines are mainly released by the adipose tissue (especially visceral fat), whereas cytokines are mainly produced by leukocytes and other cells throughout the body. We established a concept of adipokine/cytokine imbalance $\sim 15$ years ago, suggesting that the lack of anti-inflammatory mediators, e.g. adiponectin, and increased release of proinflammatory cytokines, such as interleukin (IL)-1 $\beta$, IL-6 or tumour necrosis factor (TNF)- 
$\alpha$, might result in a proinflammatory local and systemic milieu in NAFLD ${ }^{40}$. The potent antiinflammatory adipokine adiponectin comprises one of the major products of the adipose tissue, and obesity-related disorders, including NAFLD, are characterized by hypoadiponectinaemia. An expanded and dysfunctional adipose tissue, especially visceral adipose tissue, exhibits massively increased production of several proinflammatory cytokines besides decreased synthesis of certain anti-inflammatory adipokines (e.g., adiponectin) or cytokines (e.g., IL-37). Interestingly, the adipose tissue is able to contribute to at least one third of circulating IL-6 levels, a major cytokine in obesity-related disorders mainly responsible for increased plasma C-reactive protein concentrations. Many preclinical NAFLD studies have convincingly shown that neutralization of either IL-1 $\beta$ or TNF $\alpha$ abolished hepatic inflammation, steatosis and associated pathologies. What remains unclear when considering NAFLD as a systemic chronic inflammatory disorder is which compartments in the body besides adipose tissue (and the liver) might contribute to chronic inflammation. Skeletal muscle has also been proposed as a site of synthesis of various mediators i.e. myokines with autocrine, paracrine or endocrine effects ${ }^{41}$. To what extent muscle fibers contribute to the overall inflammatory load in NAFLD e.g. via production of the myokine IL- 6 is currently unclear ${ }^{42}$. Aerobic and resistance exercise are beneficial in people with NAFLD, and are accompanied by improvements of insulin resistance and decreased circulating IL-6 levels. Skeletal muscle may also play a role in NAFLD by the release of metabolically beneficial myokines, such as $\operatorname{irisin}^{43}$.

\subsection{Microbiome and NAFLD}

It is increasingly recognized that the gastrointestinal tract with its microbiota may contribute to this phenomenon ${ }^{44}$. Large clinical studies have shown that NAFLD is accompanied by a dysbiosis characterized by a bloom in certain Enterobacteriaceae, Escherichia coli and a decrease in Faecalibacterium prausnitzii ${ }^{45}$. Recent data also suggest that intestinal dysbiosis and microbiome instability exists over many years and might even precede the development of NAFLD and T2DM ${ }^{46}$. Furthermore, emerging evidence has suggested that in NAFLD bacterial components might be found in the liver ${ }^{47}$. Intestinal microbiota converts nutrients, such as choline or carnitine into trimethylamine (TMA), which is metabolized in the liver by flavin monoxygenases to TMA $N$-oxide (TMAO). Studies have now shown that TMAO is associated with CVD, and that TMAO is able to increase platelet responsiveness and thrombosis formation ${ }^{48}$. Therefore, bacterial components and the associated production of intestinal-derived metabolites might contribute to the development of liver disease in NAFLD 49 . 


\subsection{Extra-hepatic cancers in NAFLD: a role for a proinflammatory environment?}

NAFLD is associated not only with HCC, but with an excess of various other extra-hepatic cancers. Chronic inflammation is a "driving force" in many malignancies. Several inflammation-related cancers arise from the gastrointestinal tract, such as gastric cancer, pancreatic cancer, inflammatory bowel disease-related colonic cancer or liver cancer. A large biopsy-based cohort study from Sweden demonstrated that extra-hepatic cancers were the most common reason for increased mortality in people with NAFLD, followed by liverrelated and CVD-related deaths ${ }^{36}$. NAFLD is also associated with extra-hepatic cancers, such as gastrointestinal and gynaecological cancers at a higher rate as obesity itself ${ }^{34}$. A significantly higher rate of colorectal tumours has also been observed in NAFLD ${ }^{50}$, and NAFLD is characterized by significantly higher numbers of esophageal and stomach cancers. Furthermore, obesity and NAFLD are associated with a higher incidence of erosive esophagitis, Barrett's dysplasia, and esophageal adenocarcinoma ${ }^{34}$. HCC is another typical cancer associated with chronic liver inflammation caused by various aetiologies and observed in precirrhotic stages of liver disease. This tumor is strongly influenced by the immune system and is characterized by cancer-promoting inflammation exerted by the underlying disease ${ }^{51}$.

Regardless of its origin, therefore, chronic inflammation at various sites of the body may have tumour-promoting effects ${ }^{52}$. In the interplay of molecules in cancer-associated inflammation, several key factors have been identified, such as nuclear factor-kappa B (NF$k B$ ), signal transducer and activator of transcription (STAT)-3 and various proinflammatory cytokines, such as IL-1 $\beta$, IL-6, or TNF $\alpha$. Several dietary and genetic preclinical NAFLD models have shown that proinflammatory cytokines, such as IL-6, are crucially involved in liver oncogenesis ${ }^{53}$. A proinflammatory tumour-promoting environment may also be generated by lack of crucial anti-inflammatory mediators, such as adiponectin. Indeed, accumulating evidence suggests that adiponectin suppresses tumour formation at least in preclinical studies. Adiponectin also exerts anti-carcinogenic effects in vitro, by inhibiting growth of colonic cancer cells through activation of adenosine monophosphate-activated protein kinase ${ }^{54}$. Adiponectin knockout mice show not only more inflammation but also higher numbers and larger colonic tumours after injection of dextran sodium sulphate and in adiponectin KO/Apc (Min) mice ${ }^{55}$. In summary, whereas there is now evidence that NAFLD is associated with certain extra-hepatic cancers and many different mechanisms have been explored, the precise mechanisms linking chronic inflammation and cancer development with NAFLD remain uncertain.

\subsection{Proinflammatory hits targeting vessels in NAFLD}


Besides myocardial infarction and stroke, cardiac arrthymias and cardiomyopathy may all contribute to increased CVD mortality ${ }^{56}$. A proinflammatory environment in NAFLD may contribute to CVD morbidity and mortality. Proinflammatory cytokines such as macrophagederived IL-1 $\beta$ induce arrhythmias in diabetic mice ${ }^{57}$. TNF $\alpha$, IL-6 and IL-17 also have the potential to cause cardiac arrhythmias (mostly AF) in preclinical models. Increased serum IL6 and $\mathrm{C}$-reactive protein levels are correlated with a higher risk of $\mathrm{AF}^{58}$. In an experimental model, a specific NF-кB inhibitor abrogated cardiac inflammation and ameliorated arrythmogenic cardiomyopathy ${ }^{59}$. The Canakinumab Antiinflammatory Thrombosis Outcome Study (CANTOS) trial recently proved a major role for IL-1 $\beta$ in the role of atherosclerosis ${ }^{60}$ as treatment with canakinumab (i.e. an anti-IL-1 $\beta$ antibody) resulted in a significant reduction in recurrent CVD events and mortality. However, clinical interventions targeting proinflammatory cytokines are needed to provide further evidence of a causal link between chronic inflammation, CVD events and cancers associated with NAFLD.

\section{Interrelationships between NAFLD, HCC and common extra-hepatic complications} In NAFLD, liver disease per se is an independent risk factor for T2DM and CVD (and potentially CKD too), suggesting that the diseased liver is a "driving force" in the vicious circle of spiraling and worsening disease in NAFLD and T2DM"1,62. Figure 2 illustrates the "vicious spiral" of potentially worsening disease when NAFLD and T2DM co-exist, and the figure illustrates schematically, the relationships between NAFLD, T2DM, CVD, CKD, and HCC.

The liver also plays a key role in contributing to the features of metabolic syndrome (MetS $)^{63}$. The MetS includes atherogenic dyslipidaemia, increased blood pressure, dysglycaemia/T2DM, and central obesity ${ }^{63}$. In patients with newly diagnosed T2DM, the presence of MetS is independently associated with incident CVD ${ }^{64}$. Moreover, an increasing number of the individual features of MetS present at the time of diagnosis of T2DM is also associated with a linear increase in CVD risk with an almost 5-fold increase when all features of MetS are present, compared with one feature alone ${ }^{64}$. Similarly, in persons without T2DM and CVD, not only is MetS common ( $20 \%$ of subjects), but also the presence of MetS is associated with an increased risk of CVD and T2DM (in both men and women) ${ }^{65}$. Since the features of MetS are even more common in people with NAFLD (occurring in $>50 \%$ of these subjects), the presence of MetS features in those with NAFLD also potentially increases the risk of comorbidities too (such as T2DM, CVD, and CKD) that all share similar cardiometabolic risk factors ${ }^{66,67,68}$. 


\section{Diagnosis and management of extra-hepatic complications}

\subsection{Diagnosis of extra-hepatic complications}

As discussed above, people with NAFLD are at increased risk of T2DM, CVD (including atrial fibrillation), CKD and HCC. It is, therefore, important for the clinican to know how best to diagnose these related conditions. In people with NAFLD, the coexistence of MetS features and more traditional CVD risk factors, such as older age, smoking, hypertension (and/or atrial fibrillation), and increased plasma LDL-cholesterol concentration, should alert the clinician to the possibility of the presence of T2DM, CVD or CKD, as these conditions share common risk factors. For those individuals with NAFLD, who have any evidence of cirrhosis \pm portal hypertension (based on clinical features, imaging techniques or histology), there is a need for regular suveillance of $\mathrm{HCC}$ with ultrasonography ${ }^{69}$. Although a substantial proportion of NAFLD individuals with less severe liver disease can develop HCC, presently it is not recommended that this group undergo regular surveillance. Similarly, although current evidence also suggests that NAFLD increases the risk of some extra-hepatic cancers (discussed above), presently it is not recommended that screening tests are undertaken for these cancers in people with NAFLD. As our focus it to discuss extra-hepatic complications and their diagnosis and management, we have excluded further discussion of extra-hepatic cancers or HCC in this section.

\subsection{Diagnosis of T2DM, CKD and atrial fibrillation and assessment of CVD risk}

Although a false positive diagnosis of T2DM is unlikely to cause as much anxiety as a false positive diagnosis of cancer, it is important to consider the effect of a diagnosis of T2DM on the quality of life and health-related behaviour. That said, in recent years it has become easy to screen a patient at risk of T2DM, with measurement of haemoglobin A1c ( $\mathrm{HbA} 1 \mathrm{c})$ and a concentration of $\geq 43$ or $\geq 48 \mathrm{mmol} / \mathrm{mol}$ is indicative of prediabetes or T2DM, respectively. The current American Diabetes Association (ADA) guidelines recommend that $\mathrm{HbA} 1 \mathrm{c}$ testing should be undertaken in all individuals $\geq 45$ years and in those of any age with overweight or obesity, with one or more risk factors for $\mathrm{T}_{2} \mathrm{DM}^{70}$. $\mathrm{HbA} 1 \mathrm{c}$ has many advantages over and above previous diagnostic tests for T2DM that involved measurement of fasting plasma glucose or an oral glucose tolerance test. These advantages include greater convenience (fasting not required), greater pre-analytical stability, and fewer day-to-day perturbations during stress, diet, or illness. However, these advantages are somewhat offset by the lower sensitivity of $\mathrm{HbA} 1 \mathrm{c}$ at the designated cut-point and greater cost. The $\mathrm{HbA} 1 \mathrm{c}$ test, with a diagnostic threshold of $48 \mathrm{mmol} / \mathrm{mol}$, diagnoses only $\sim 30 \%$ of T2DM cases identified collectively using $\mathrm{HbA} 1 \mathrm{c}$, fasting plasma glucose, or 2-hour glucose concentrations (during an oral glucose tolerance test), according to National Health and Nutrition Examination Survey-III data ${ }^{71}$. Despite these caveats for individuals at risk of T2DM, such as those with 
NAFLD who have a normal initial diagnositc test for T2DM, the ADA guidelines recommend that the $\mathrm{HbA} 1 \mathrm{c}$ test should be repeated at a minimum of a 3-year interval ${ }^{70}$. Similar to current standard practice that is common place amongst primary care physicans and diabetologists for patients with a diagnosis of T2DM, we would advocate that measurement of adiposity measures, blood pressure, and pulse rate, the assessment of the possible presence of MetS, atrial fibrillation and CKD, as well as the use of a CVD risk calculator to assess the individual's CVD risk should be undertaken in all individuals with NAFLD. The presence of CKD can be assessed by measurement of eGFR and urinary albumin excretion and a level of eGFR $\geq 90 \mathrm{mls} / \mathrm{min}$ together with a urinary albumin excretion $<30 \mathrm{mg} / \mathrm{g}$ is considered normal. There are five GFR stages/categories in CKD, and when CKD stage 3 to 5 exists, defined by a decreased level of eGFR, it is important to note that there is a further (at least $\geq 50 \%$ ) increase in 10-year CVD risk.

Although there are over 100 CVD risk prediction models, and various CVD risk calculators, only about 25 of these models/calculators have been externally validated ${ }^{72}$. It should be noted that CVD risk calculators are only able to generate approximate estimates of the global CVD risk. Calculators vary according to the database they are derived from, choice of clinical endpoints and risk interval duration on which the estimate is based. Addition of other risk factors does little to improve risk prediction performance, with the exception perhaps of the coronary artery calcium score (CACS) (see below); although this still requires further study $^{72}$. Furthermore, whether addition of NASH, either with or without liver fibrosis, to a CVD risk algorithm, improves CVD risk prediction is uncertain. That said, the use of a CVD risk calculator without entering specific information about liver disease in individuals with NAFLD, is better than not estimating CVD risk. Thus, we recommend that healthcare professionals should use a CVD risk calculator based on a CVD risk prediction model that is derived from, or is calibrated for, a population similar to the patient in question ${ }^{72}$. In general, current management of the CVD risk in people with NAFLD widely overlaps with the guidelines for the treatment of CVD risk factors, which are adopted for the general population.

It is known that the CACS is a strong predictor of CVD event rates in the general population for at least up to $\sim 15$ years of follow-up ${ }^{73,74}$, and assessment of the CACS may improve CVD risk prediction, particularly in those patients where there is uncertainty about individual CVD risk. The CACS is obtained from high-resolution computed tomography of the chest and is usually presented as a number called Agatston score. That score reflects the total area of coronary calcium deposits and the density of the calcium. When calcium is present, the higher the score, the higher is the CVD risk. A score of 100 to 300 means moderate 
coronary plaque deposits and a score greater than 300 is a sign of high CVD risk, particularly in a younger person. Comparing both the FRS and the CACS, there is substantive risk reclassification (that is, net reclassification index $>0.20$ ) in patients with CACS and CVD risk factors beyond age and other traditional risk factors ${ }^{74}$. Additionally, a CACS of 400 or greater had a 15 -year all-cause mortality rate that exceeded $20 \%$, confirming the importance of length of follow-up in defining substantively higher-risk status ${ }^{74}$.

One obvious problem with multislice computed tomography is the projected risk of cancer, after exposure to ionizing radiation, and it has been estimated that the cancer risk is $\sim 12$ cancers out of 10,000 screened patients ${ }^{75}$. Additionally, although CACS is a well-established assessment for risk stratifying asymptomatic patients for overall CVD or coronary events; the prognostic value of CACS for incident ischaemic stroke is less clear. However, CACS may also be useful for assesing risk of stroke as recently, in a meta-analysis of 13,262 asymptomatic patients without evidence of apparent CVD, the presence and severity of CACS was associated with increased risk of incident ischaemic stroke during a median follow up of 7.2 years $^{76}$. The 2018 ACC/AHA cholesterol guidelines suggest that CACS testing should be considered in individuals $40-75$ years of age without diabetes and with a plasma LDL-cholesterol level between 70 and $189 \mathrm{mg} / \mathrm{dl}$ at a 10 -year CVD risk of $\geq 7.5 \%$ to $<20 \%$ (i.e., intermediate CVD risk group), particularly if there is uncertain about whether medical treatment should be instigated ${ }^{77}$. Thus, in summary, for asymptomatic patients, calculation of CACS may refine CVD risk prediction and guide patient management and this benefit may offset the minimal projected risk of cancer after exposure to ionizing radiation ${ }^{75}$.

\subsection{Management and treatment of increased CVD risk, T2DM and CKD}

Statin therapy is known to be safe in people with NAFLD. According to the above-mentioned guidelines $^{77}$, a CACS score of 1 to 99 in patients favors statin therapy, especially in those $\geq 55$ years of age. For any patient, if the CACS score is $\geq 100$, statin treatment is indicated unless otherwise agreed by the outcome of clinician-patient discussion. In those patients with evidence of CACS, the challenge is to decide which patients require further investigation and which patients require intensive medical management alone and it is important in this situation to take adivce from cardiological colleagues. The vast majority of asymptomatic patients with elevated CACS scores do not need any further testing. However, these patients do require aggressive medical treatment, e.g. low-dose aspirin, angiotensin converting enzyme inhibitor or angiotensin II receptor blocker, and lifestyle intervention ${ }^{78}$.

Lifestyle measures, such as increased physical activity and weight reduction, are effective not only in ameliorating the early stages of liver disease in NAFLD, but are also effective in 
improving glycaemic control, resolution of T2DM and also in improving CVD and CKD risk factors. Several randomized clinical trials and a recent meta-analysis have investigated the role of physical activity, independent of dietary intervention, in people with NAFLD ${ }^{79,80}$. A meta-analysis of 16 randomized controlled trials with a total of $\sim 700$ individuals with NAFLD showed that exercise without dietary intervention improved liver fat content, as assessed by magnetic resonance-based techniques ${ }^{79}$. Lifestyle measures are also effective in improving many of the shared cardio-metabolic risk factors that exist between NAFLD, T2DM and CVD. For example, weight reduction is also effective for improving coexisting cardiometabolic risk factors, such as increased blood pressure, atherogenic dyslipidaemia, and for decreasing plasma glucose concentrations and effecting remission of T2DM ${ }^{81-84}$. How much weight reduction should be advocated for a particular patient will in part depend on the level of overweight or obesity. However, weight reduction of $\geq 10 \%$ induces NASH resolution, as well as improves liver fibrosis by at least one histological stage ${ }^{81}$, and for most patients this amount of weigth loss should be advocated. However, weight reduction of $\geq 10 \%$ can be difficult to achieve and lower amounts of weight loss (e.g. from $5 \%$ to $10 \%$ ) can also improve hepatic steatosis and NASH, as assessed by the histological NAFLD activity score ${ }^{81}$. Consequently, the EASL-EASO-EASD, the American Association for the Study of Liver Diseases (AASLD) and the National Institute for Health and Care Excellence (NICE) practice guidelines for management of NAFLD emphasize that in overweight or obese individuals with NAFLD, 5-10\% weight loss is the goal of most lifestyle interventions ${ }^{85-87}$ and this amount of weight loss will also have favourable effects on cardio-metabolic risk factors. It is important to note that nonobese individuals with NAFLD can also achieve improvements in liver disease with weight reduction of 3-10\%, and nonobese individuals are also more likely than obese patients to maintain weight loss (and normal serum liver enzyme levels) over time. It is also sensible to recommend that people with NAFLD should avoid alcohol consumption, potentially hepatotoxic drugs, cigarette smoking and high sucrose/fructosecontaining drinks and food ${ }^{85,86}$.

In the US, the American College of Cardiology/American Heart Association guidelines for the primary prevention in adults to reduce the risk of major atherosclerotic CVD events recommend that adults should be categorized into low ( $<5 \%$ ), borderline ( 5 to $<7.5 \%$ ), intermediate ( $\geq 7.5$ to $<20 \%$ ), or high ( $\geq 20 \%$ ) 10-year CVD risk. Statin treatment to attenuate risk is advocated in middle-aged people at borderline CVD risk ${ }^{88}$. Although in Europe the threshold for medical intervention is not so low, it should be noted that the threshold of 10year CVD risk at which healthcare professionals should advocate medical intervention to lower that increased risk remains uncertain. As mentioned above, the assessment of CACS may help refine CVD risk prediction where there is uncertainty ${ }^{77}$, e.g. a CACS score of 1 to 
99 favours statin therapy, especially in those $\geq 55$ years of age. For patients of any age, if the CACS score is $\geq 100$, statin treatment is indicated unless otherwise agreed by the outcome of a clinician-patient discussion. In those patients with evidence of CACS, the challenge is to decide which patients require further investigation and which patients require intensive medical management alone and it is important in this situation to take adivce from cardiological colleagues. The large majority of asymptomatic individuals with elevated CACS scores do not need any further testing. However, these subjects do require aggressive medical treatment, e.g. low-dose aspirin, angiotensin converting enzyme inhibitor or angiotensin II receptor blocker, and lifestyle intervention ${ }^{78}$.

Although metformin treatment is the first-line oral treatment of choice in people with T2DM, there is not convincing evidence that metformin treatment confers benefit on liver disease in NAFLD. In contrast, although pioglitazone has become the 'forgotten cost-effective, cardioprotective drug for T2DM'89 that not only has a durable action to lower blood glucose and decrease progression from impaired glucose tolerance to T2DM, treatment with pioglitazone also decreases risk of myocardial infarction and ischaemic stroke. Despite having some recognized side effects, treatment with pioglitazone also produces benefit in $\mathrm{NASH}$ in patients with and without T2DM ${ }^{90,91}$. Thus, given the recommendations from the USA, Europe and the UK that pioglitazone treatment should be considered in $\mathrm{NASH}^{85-87}$, this drug is often not used in individuals with $\mathrm{NASH}$, either with, or without T2DM.

Liver histological improvement depends on the amount of weight reduction rather than the intervention used to achieve this goal ${ }^{81}$. For example, drugs such as orlistat and glucagonlike peptide-1 (GLP-1) receptor agonists, such as liraglutide and semaglutide, facilitate weight reduction and also are associated with histological resolution of NASH and, in some cases, fibrosis regression $81,92,93$.

In Figure $\mathbf{3}$ we have illustrated schematically a potential approach to diagnosing and managing extra-hepatic complications of NAFLD, such as T2DM, CVD, CKD, and HCC.

\section{Areas of uncertainty}

We believe that combating the growing burden of NAFLD and its multisystem complications extending beyond the liver will require the setting up a multi-disciplinary team approach to embrace collaborative ways of working to deliver holistic patient-centred care and management for patients with this condition. Whilst care of the person with advanced liver disease in NAFLD has traditionally been the remit of the hepatologist, liasing with the primary care physician, many of these individuals with less severe liver disease are 
frequently patient attendees in other clinics, e.g. diabetes, cardiology, renal, run by physicians who know little about NAFLD and its complications and management. Indeed, in this Review, we call for a more person-centered, multidisciplinary and holistic approach involving the healthcare professionals as shown in Figure 4, with a view to better advocacy for this patient group and the potential for earlier diagnosis and management of liver-related and extra-hepatic complications to improve the patient's outcomes. As to how best achieve this goal remains uncertain. We recommend further work is needed to decide the best structures of healthcare delivery for people with NAFLD. It is likely that this healthcare delivery will be different across individual nation states, because of the different structural organization of nations' healthcare systems.

Currently, it remains uncertain whether individuals with non-cirrhotic NASH should undergo HCC surveillance. For those who have T2DM and NAFLD, metformin is the first-line oral treatment for hyperglycaemia. However, current evidence does not support a beneficial effect of metformin treatment on liver disease and further studies are needed testing the effect of metformin on risk of HCC. Although there is good evidence that CACS assessment in the general population can be used to improve CVD risk prediction, further evidence is needed in people with NAFLD who may have features of MetS, but who do not have T2DM. Although statins are safe in individuals who have NAFLD, some individuals with NAFLD have serum transaminase concentrations that are very high (>2-3 times the upper limit of normal) and better evidence is needed regarding surveillance and the long-term effects of statins on the liver in this subgroup of individuals. There is increasing interest in the potential benefit of long-acting injectable GLP-1 receptor agonists in NASH, not least with the recently publication of favourable data with semaglutide ${ }^{93}$ and because these glucose-lowering agents facilitate weight reduction and decrease CVD risk. However, it is uncertain whether any benefit on the liver in NASH is independent of weight reduction. Additionally, recent favourable data from a 24-week treatment with the pan-peroxisomal proliferator activated receptor (PPAR) lanifibranor - reported at the 2020 AASLD meeting ${ }^{94}$ - has again placed the focus on the role of PPAR agonists in treatment of NASH and further data is required with this drug from ongoing phase-3 controlled trials.

\section{Conclusions}

This Review outlines the strong association between the presence and severity of NAFLD and the risk of developing multiple extra-hepatic complications. Despite the growing evidence that links NAFLD with T2DM, CVD, CKD, and certain types of extra-hepatic cancers, it remains to be definitively established whether a causal association also exists. 
It should be noted that existing guidelines from non-hepatological societies do not advocate screening for NAFLD and its liver-related complications in people with CVD or CKD or T2DM, making the liver a potentially neglected organ and meaning that chronic disease progression to cirrhosis might be largely undetected. Conversely, existing guidelines from hepatological societies recommend screening for T2DM and CVD, but do not recommend any specific screening for CKD or extra-hepatic cancers nor exactly define the tests to do for the screening for CVD complications. Currently, all individuals with NAFLD should undergo similar screening programs for CVD, T2DM, CKD, and extra-hepatic cancers to those usually adopted for individuals without NAFLD of comparable age and sex.

We also call for better advocacy and a multi-disciplinary team approach for people with NAFLD. Improved collaborative ways of working amongst the healthcare professionals caring for this patient group, will hopefully achieve earlier diagnosis and better management, not only of liver disease, but also the extra-hepatic complications of this multi-system disease.

Disclosure statement: The Authors have no conflicts of interest to disclose.

Acknowledgments: CDB is supported in part by grants from the Southampton National Institute for Health Research Biomedical Research Centre. GT is supported in part by grants from the University School of Medicine of Verona, Verona, Italy. HT is supported by the excellence initiative VASCage (Centre for Promoting Vascular Health in the Ageing Community), an R\&D K-Centre (COMET program - Competence Centers for Excellent Technologies) funded by the Austrian Ministry for Transport, Innovation and Technology, the Austrian Ministry for Digital and Economic Affairs and the federal states Tyrol, Salzburg and Vienna.

Search strategy and selection criteria: References for this clinical narrative Review were identified through searches of PubMed with the search terms "nonalcoholic fatty liver disease" OR "NAFLD" AND “incident diabetes", "incident cardiovascular disease”, "incident chronic kidney disease", OR "incident extrahepatic cancers", from the inception date to December 6, 2020. Articles were also identified through searches of the authors' own files. Only papers published in English were reviewed. The final reference list was generated on the basis of originality and relevance to the broad scope of this Review. 


\section{Figure Legends}

Figure 1. NAFLD-related effects on development of cardiovascular disease, type 2 diabetes mellitus and other extra-hepatic diseases.

NAFLD may promote the development of type 2 diabetes mellitus, accelerated coronary atherosclerosis, arrhythmias (mostly permanent atrial fibrillation), chronic kidney disease, and some types of extra-hepatic cancers. NAFLD, especially in its more severe histological forms (NASH with varying amounts of liver fibrosis) exacerbates hepatic/systemic insulin resistance and compensatory hyperinsulinaemia, promotes atherogenic dyslipidaemia, induces hypertension, and causes the synthesis of a myriad of pro-atherogenic, procoagulant and pro-inflammatory mediators that may contribute to the development of T2DM, CVD, and other extra-hepatic complications.

Figure 2. Relationships between NAFLD and T2DM, MetS, CVD, CKD or HCC. This schematic figure illustrates the relationships between NAFLD, type 2 diabetes mellitus (T2DM) and metabolic syndrome (MetS), cardiovascular disease (CVD), chronic kidney disease (CKD), and hepatocellular carcinoma (HCC). Both T2DM and NAFLD are associated with features of the MetS. The features of MetS are central obesity, atherogenic dyslipidaemia, hyperglycaemia (increased plasma triglycerides and small dense LDL-C, and decreased HDL-C concentrations), and hypertension, which are established CVD risk factors. T2DM, CVD and CKD share risk factors and, therefore, these conditions frequently occur together. NAFLD is an independent risk factor for T2DM, CVD and CKD. T2DM is an important risk factor for both CVD and CKD, and therefore with the development of T2DM in patients with NAFLD there is a further increase in risk of CVD and CKD, as well as a worsening of liver disease (advanced fibrosis and cirrhosis) that also increases risk of HCC. Recent evidence suggests that development of advanced fibrosis with NAFLD also further increases risk of CVD.

Figure 3. Flow diagram describing a pragmatic approach to the diagnosis of T2DM, CVD, CKD, and HCC, together with initial management approaches to treating T2DM, CVD and CKD as extra-hepatic complications of NAFLD.

This flow diagram emphasizes the need to assess risk of T2DM, CVD, CKD and HCC in all patients with diagnosed NAFLD. The diagram shows how to diagnose T2DM and CKD and how to assess risk of CVD. In people with increased CVD risk who are unable to take statins, consideration is necessary of whether further non-invasive investigation of potential CVD is required. Where high-resolution computed tomography (CT) is available, assessment of the coronary artery calcium scoring (CACS) offers a quick, but relatively expensive, investigation for refining CVD risk prediction and for identifying probable CVD. Where CVD score is increased (e.g. $\geq 90^{\text {th }}$ centile adjusted for age and sex or the score is high e.g. $>300$ ), referral to Cardiology should be considered as patients may be asymptomatic but have reversible cardiac ischaemia. Such individuals may require not only high-dose statin treatment, but also low-dose aspirin and angiotensin converting enzyme inhibitor (ACE-i) or angiotensin II receptor blocker treatment. Although statins are now known to be safe in people with NAFLD, for those individuals at high CVD risk (determined by use of a CVD risk algorithm, such as the Framingham Risk Score or QRISK3 score), some individuals will not be able to tolerate statin treatment. In these individuals other LDL-C lowering treatment may be required, such ezetimibe or proprotein convertase subtilisin/kexin type-9 (PCSK9) monoclonal antibody treatment, and further specialist advice may be sought. In people with T2DM, metformin treatment should be first-line oral hypoglycaemic drug, although current evidence shows that metformin does specifically benefit liver disease in NAFLD. For T2DM individuals with inadequate glycaemic control (e.g. $\mathrm{HbA} 1 \mathrm{c}>55 \mathrm{mmol} / \mathrm{mol}$ ), there should be a low threshold for further intensification of treatment with consideration given to agents, such 
as pioglitazone, long-acting injectable glucagon-like peptide-1 (GLP-1) receptor agonists, and sodium-glucose co-transporter-2 (SGLT-2) inhibitors. Although there is not presently convincing evidence of benefit to the liver with the later, SGLT-2 inhibitors may be considered specifically in individuals with evidence of CKD. In all individuals, lifestyle advice should be given and this will include advocating 150-200 min/week of physical activity/exercise, a Mediterranean-style of diet; a goal of $10 \%$ weight reduction with overweight or obesity facilitated by a calorie-restricted diet, consideration of treatment with glucagon-like peptide 1 (GLP-1) receptor agonists for weight loss/T2DM and, possibly, bariatric surgery for those with severe obesity. It is also important to emphasize limiting saturated fat intake, red/processed meats, refined carbohydrates and sugar-sweetened drinks together with smoking cessation, if relevant.

Figure 4. Person-centered, multidisciplinary and holistic approach to individuals with NAFLD.

The increasing complexity of NAFLD underscores the importance of a person-centered multidisciplinary team approach to the diagnosis, treatment, and supportive care of these patients. NAFLD should become a target for the prevention and treatment of T2DM, CVD, CKD and some extra-hepatic cancers. NAFLD is, therefore, a clinical condition that deserves greater attention from gastroenterologists/hepatologists, diabetologists/endocrinologists, cardiologists, nephrologists and internists, as well as general practitioners and dietitians. 


\section{References}

1. Tsochatzis EA, Newsome PN. Non-alcoholic fatty liver disease and the interface between primary and secondary care. The lancet Gastroenterology \& hepatology 2018; 3(7): 509-17.

2. Mantovani A, Scorletti E, Mosca A, Alisi A, Byrne CD, Targher G. Complications, morbidity and mortality of nonalcoholic fatty liver disease. Metabolism 2020; 111s: 154170.

3. Paik JM, Golabi P, Younossi Y, Mishra A, Younossi ZM. Changes in the Global Burden of Chronic Liver Diseases From 2012 to 2017: The Growing Impact of NAFLD. Hepatology 2020; 72(5): 1605-16.

4. Byrne CD, Targher G. NAFLD: A multisystem disease. J Hepatol 2015; 62(1S): S47-S64.

5. Younossi ZM, Blissett D, Blissett R, et al. The economic and clinical burden of nonalcoholic fatty liver disease in the United States and Europe. Hepatology 2016; 64(5): 1577-86.

6. Eslam M, Newsome PN, Sarin SK, et al. A new definition for metabolic dysfunction-associated fatty liver disease: An international expert consensus statement. J Hepatol 2020; 73(1): 202-9.

7. Eslam M, Sanyal AJ, George J. MAFLD: A Consensus-Driven Proposed Nomenclature for Metabolic Associated Fatty Liver Disease. Gastroenterology 2020; 158(7): 1999-2014.e1.

8. Eslam M, Sarin SK, Wong VW, et al. The Asian Pacific Association for the Study of the Liver clinical practice guidelines for the diagnosis and management of metabolic associated fatty liver disease. Hepatology international 2020.

9. Mendez-Sanchez N, Arrese M, Gadano A, et al. The Latin American Association for the Study of the Liver (ALEH) position statement on the redefinition of fatty liver disease. The lancet Gastroenterology \& hepatology 2021; 6(1): 65-72. 
10. Shiha G, Alswat K, Al Khatry M, et al. Nomenclature and definition of metabolic-associated fatty liver disease: a consensus from the Middle East and north Africa. The lancet Gastroenterology \& hepatology 2021; 6(1): 57-64.

11. Shiha G, Korenjak M, Eskridge W, et al. Redefining fatty liver disease: an international patient perspective. The lancet Gastroenterology \& hepatology 2021; 6(1): 73-9.

12. Lin S, Huang J, Wang M, et al. Comparison of MAFLD and NAFLD diagnostic criteria in real world. Liver Int 2020; 40(9): 2082-9.

13. Yamamura S, Eslam M, Kawaguchi T, et al. MAFLD identifies patients with significant hepatic fibrosis better than NAFLD. Liver Int 2020.

14. Lee H, Lee YH, Kim SU, Kim HC. Metabolic Dysfunction-Associated Fatty Liver Disease and Incident Cardiovascular Disease Risk: A Nationwide Cohort Study. Clinical gastroenterology and hepatology: the official clinical practice journal of the American Gastroenterological Association 2020.

15. Sun DQ, Jin Y, Wang TY, et al. MAFLD and risk of CKD. Metabolism 2020; 115: 154433.

16. Alemany-Pagès $\mathrm{M}$, Moura-Ramos $\mathrm{M}$, Araújo $\mathrm{S}$, et al. Insights from qualitative research on NAFLD awareness with a cohort of T2DM patients: time to go public with insulin resistance? BMC Public Health 2020; 20(1): 1142.

17. Wieland AC, Mettler P, McDermott MT, Crane LA, Cicutto LC, Bambha KM. Low awareness of nonalcoholic fatty liver disease among patients at high metabolic risk. Journal of clinical gastroenterology 2015; 49(1): e6-e10.

18. Mantovani A, Nascimbeni F, Lonardo A, et al. Association Between Primary Hypothyroidism and Nonalcoholic Fatty Liver Disease: A Systematic Review and Meta-Analysis. Thyroid : official journal of the American Thyroid Association 2018; 28(10): 1270-84.

19. Lonardo A, Mantovani A, Lugari S, Targher G. NAFLD in Some Common Endocrine Diseases: Prevalence, Pathophysiology, and Principles of Diagnosis and Management. International journal of molecular sciences 2019; 20(11). 
20. Targher G, Byrne CD, Lonardo A, Zoppini G, Barbui C. Non-alcoholic fatty liver disease and risk of incident cardiovascular disease: A meta-analysis. J Hepatol 2016; 65(3): 589-600.

21. Targher G, Byrne CD, Tilg H. NAFLD and increased risk of cardiovascular disease: clinical associations, pathophysiological mechanisms and pharmacological implications. Gut 2020; 69(9): 1691705.

22. Taylor RS, Taylor RJ, Bayliss S, et al. Association Between Fibrosis Stage and Outcomes of Patients With Nonalcoholic Fatty Liver Disease: A Systematic Review and Meta-Analysis. Gastroenterology 2020; 158(6): 1611-25.e12.

23. Henson JB, Simon TG, Kaplan A, Osganian S, Masia R, Corey KE. Advanced fibrosis is associated with incident cardiovascular disease in patients with non-alcoholic fatty liver disease. Aliment Pharmacol Ther 2020; 51(7): 728-36.

24. Sinn DH, Cho SJ, Gu S, et al. Persistent Nonalcoholic Fatty Liver Disease Increases Risk for Carotid Atherosclerosis. Gastroenterology 2016; 151(3): 481-8.e1.

25. Sinn DH, Kang D, Chang Y, et al. Non-alcoholic fatty liver disease and progression of coronary artery calcium score: a retrospective cohort study. Gut 2017; 66(2): 323-9.

26. Anstee QM, Mantovani A, Tilg H, Targher G. Risk of cardiomyopathy and cardiac arrhythmias in patients with nonalcoholic fatty liver disease. Nat Rev Gastroenterol Hepatol 2018; 15(7): 425-39.

27. Cai X, Zheng S, Liu Y, Zhang Y, Lu J, Huang Y. Nonalcoholic fatty liver disease is associated with increased risk of atrial fibrillation. Liver Int 2020; 40(7): 1594-600.

28. Hung CS, Tseng PH, Tu CH, et al. Nonalcoholic Fatty Liver Disease Is Associated With QT Prolongation in the General Population. Journal of the American Heart Association 2015; 4(7).

29. Mantovani A, Petracca G, Beatrice G, Tilg H, Byrne CD, Targher G. Non-alcoholic fatty liver disease and risk of incident diabetes mellitus: an updated meta-analysis of 501022 adult individuals. Gut 2020. 
30. Sung KC, Wild SH, Byrne CD. Resolution of fatty liver and risk of incident diabetes. JClinEndocrinolMetab 2013; 98(9): 3637-43.

31. Yamazaki H, Tsuboya T, Tsuji K, Dohke M, Maguchi H. Independent Association Between Improvement of Nonalcoholic Fatty Liver Disease and Reduced Incidence of Type 2 Diabetes. Diabetes Care 2015; 38(9): 1673-9.

32. Mantovani A, Petracca G, Beatrice G, et al. Non-alcoholic fatty liver disease and risk of incident chronic kidney disease: an updated meta-analysis. Gut 2020.

33. Mantovani A, Dauriz M, Byrne CD, et al. Association between nonalcoholic fatty liver disease and colorectal tumours in asymptomatic adults undergoing screening colonoscopy: a systematic review and meta-analysis. Metabolism 2018; 87: 1-12.

34. Allen AM, Hicks SB, Mara KC, Larson JJ, Therneau TM. The risk of incident extrahepatic cancers is higher in non-alcoholic fatty liver disease than obesity - A longitudinal cohort study. J Hepatol 2019; 71(6): 1229-36.

35. Wang Z, Zhao X, Chen S, et al. Associations Between Nonalcoholic Fatty Liver Disease and Cancers in a Large Cohort in China. Clinical gastroenterology and hepatology : the official clinical practice journal of the American Gastroenterological Association 2020.

36. Simon TG, Roelstraete B, Khalili H, Hagström H, Ludvigsson JF. Mortality in biopsy-confirmed nonalcoholic fatty liver disease: results from a nationwide cohort. Gut 2020.

37. Sheka AC, Adeyi O, Thompson J, Hameed B, Crawford PA, Ikramuddin S. Nonalcoholic Steatohepatitis: A Review. JAMA 2020; 323(12): 1175-83.

38. Trépo E, Valenti L. Update on NAFLD genetics: From new variants to the clinic. J Hepatol 2020; 72(6): 1196-209.

39. Hotamisligil GS. Inflammation, metaflammation and immunometabolic disorders. Nature 2017; 542(7640): 177-85. 
40. Tilg H, Hotamisligil GS. Nonalcoholic fatty liver disease: Cytokine-adipokine interplay and regulation of insulin resistance. Gastroenterology 2006; 131(3): 934-45.

41. Pedersen BK, Febbraio MA. Muscles, exercise and obesity: skeletal muscle as a secretory organ. Nat Rev Endocrinol 2012; 8(8): 457-65.

42. Pedersen BK, Fischer CP. Beneficial health effects of exercise--the role of IL-6 as a myokine. Trends in pharmacological sciences 2007; 28(4): 152-6.

43. Metwally M, Bayoumi A, Romero-Gomez M, et al. A polymorphism in the Irisin-encoding gene (FNDC5) associates with hepatic steatosis by differential miRNA binding to the 3'UTR. J Hepatol 2019; 70(3): 494-500.

44. Tilg H, Zmora N, Adolph TE, Elinav E. The intestinal microbiota fuelling metabolic inflammation. Nat Rev Immunol 2020; 20(1): 40-54.

45. Loomba R, Seguritan V, Li W, et al. Gut Microbiome-Based Metagenomic Signature for Noninvasive Detection of Advanced Fibrosis in Human Nonalcoholic Fatty Liver Disease. Cell Metab 2019; 30(3): 607.

46. Frost F, Kacprowski T, Ruhlemann M, et al. Long-term instability of the intestinal microbiome is associated with metabolic liver disease, low microbiota diversity, diabetes mellitus and impaired exocrine pancreatic function. Gut 2020.

47. Sookoian S, Salatino A, Castano GO, et al. Intrahepatic bacterial metataxonomic signature in non-alcoholic fatty liver disease. Gut 2020; 69(8): 1483-91.

48. Wang Z, Klipfell E, Bennett BJ, et al. Gut flora metabolism of phosphatidylcholine promotes cardiovascular disease. Nature 2011; 472(7341): 57-63.

49. Jennison E, Byrne CD. The role of the gut microbiome and diet in the pathogenesis of nonalcoholic fatty liver disease. Clinical and molecular hepatology 2020. 
50. Wong VW, Wong GL, Tsang SW, et al. High prevalence of colorectal neoplasm in patients with non-alcoholic steatohepatitis. Gut 2011; 60(6): 829-36.

51. Zoller H, Tilg H. Nonalcoholic fatty liver disease and hepatocellular carcinoma. Metabolism 2016; 65(8): 1151-60.

52. Mantovani A, Allavena P, Sica A, Balkwill F. Cancer-related inflammation. Nature 2008; 454(7203): 436-44.

53. Park EJ, Lee JH, Yu GY, et al. Dietary and genetic obesity promote liver inflammation and tumorigenesis by enhancing IL-6 and TNF expression. Cell 2010; 140(2): 197-208.

54. Kim AY, Lee YS, Kim KH, et al. Adiponectin represses colon cancer cell proliferation via AdipoR1- and -R2-mediated AMPK activation. Mol Endocrinol 2010; 24(7): 1441-52.

55. Mutoh M, Teraoka N, Takasu S, et al. Loss of adiponectin promotes intestinal carcinogenesis in Min and wild-type mice. Gastroenterology 2011; 140(7): 2000-8, 8 e1-2.

56. Stahl EP, Dhindsa DS, Lee SK, Sandesara PB, Chalasani NP, Sperling LS. Nonalcoholic Fatty Liver Disease and the Heart: JACC State-of-the-Art Review. J Am Coll Cardiol 2019; 73(8): 948-63.

57. Monnerat G, Alarcon ML, Vasconcellos LR, et al. Macrophage-dependent IL-1beta production induces cardiac arrhythmias in diabetic mice. Nat Commun 2016; 7: 13344.

58. $\mathrm{Wu} \mathrm{N}, \mathrm{Xu} \mathrm{B}, \mathrm{Xiang} \mathrm{Y}$, et al. Association of inflammatory factors with occurrence and recurrence of atrial fibrillation: a meta-analysis. Int J Cardiol 2013; 169(1): 62-72.

59. Chelko SP, Asimaki A, Lowenthal J, et al. Therapeutic Modulation of the Immune Response in Arrhythmogenic Cardiomyopathy. Circulation 2019; 140(18): 1491-505.

60. Ridker PM, Everett BM, Thuren T, et al. Antiinflammatory Therapy with Canakinumab for Atherosclerotic Disease. N Engl J Med 2017; 377(12): 1119-31. 
61. Yki-Järvinen H. Non-alcoholic fatty liver disease as a cause and a consequence of metabolic syndrome. The Lancet Diabetes \& Endocrinology 2014; 2(11): 901-10.

62. Wainwright P, Byrne CD. Bidirectional Relationships and Disconnects between NAFLD and Features of the Metabolic Syndrome. International journal of molecular sciences 2016; 17(3): 367.

63. Alberti KG, Eckel RH, Grundy SM, et al. Harmonizing the metabolic syndrome: a joint interim statement of the International Diabetes Federation Task Force on Epidemiology and Prevention; National Heart, Lung, and Blood Institute; American Heart Association; World Heart Federation; International Atherosclerosis Society; and international association for the Study of Obesity. Circulation 2009; 120(16): 1640-5.

64. Guzder RN, Gatling W, Mullee MA, Byrne CD. Impact of metabolic syndrome criteria on cardiovascular disease risk in people with newly diagnosed type 2 diabetes. Diabetologia 2006; 49(1): 49-55.

65. Wilson PW, D'Agostino RB, Parise H, Sullivan L, Meigs JB. Metabolic syndrome as a precursor of cardiovascular disease and type 2 diabetes mellitus. Circulation 2005; 112(20): 3066-72.

66. Gastaldelli A, Cusi K. From NASH to diabetes and from diabetes to NASH: Mechanisms and treatment options. JHEP Reports 2019; 1(4): 312-28.

67. Targher G, Byrne CD. A Perspective on Metabolic Syndrome and Nonalcoholic Fatty Liver Disease. Metab Syndr Relat Disord 2015; 13(6): 235-8.

68. Francque S, De Maeght S, Adler M, et al. High prevalence of advanced fibrosis in association with the metabolic syndrome in a Belgian prospective cohort of NAFLD patients with elevated ALT. Results of the Belgian NAFLD registry. Acta Gastroenterol Belg 2011; 74(1): 9-16.

69. Plaz Torres MC, Bodini G, Furnari M, et al. Surveillance for Hepatocellular Carcinoma in Patients with Non-Alcoholic Fatty Liver Disease: Universal or Selective? Cancers 2020; 12(6).

70. 2. Classification and Diagnosis of Diabetes: $<\mathrm{em}>$ Standards of Medical Care in Diabetes2020</em>. Diabetes Care 2020; 43(Supplement 1): S14-S31. 
71. Cowie CC, Rust KF, Byrd-Holt DD, et al. Prevalence of diabetes and high risk for diabetes using A1C criteria in the U.S. population in 1988-2006. Diabetes Care 2010; 33(3): 562-8.

72. Allan GM, Garrison S, McCormack J. Comparison of cardiovascular disease risk calculators. Current opinion in lipidology 2014; 25(4): 254-65.

73. Budoff MJ, Shaw LJ, Liu ST, et al. Long-term prognosis associated with coronary calcification: observations from a registry of 25,253 patients. J Am Coll Cardiol 2007; 49(18): 1860-70.

74. Shaw LJ, Giambrone AE, Blaha MJ, et al. Long-Term Prognosis After Coronary Artery Calcification Testing in Asymptomatic Patients: A Cohort Study. Ann Intern Med 2015; 163(1): 14-21.

75. Smith-Bindman R, Lipson J, Marcus R, et al. Radiation dose associated with common computed tomography examinations and the associated lifetime attributable risk of cancer. Arch Intern Med 2009; 169(22): 2078-86.

76. Chaikriangkrai K, Jhun HY, Palamaner Subash Shantha G, et al. Coronary artery calcium score as a predictor for incident stroke: Systematic review and meta-analysis. Int J Cardiol 2017; 236: 473-7.

77. Grundy SM, Stone NJ, Bailey AL, et al. 2018 AHA/ACC/AACVPR/AAPA/ABC/ACPM/ADA/AGS/APhA/ASPC/NLA/PCNA Guideline on the Management of Blood Cholesterol: A Report of the American College of Cardiology/American Heart Association Task Force on Clinical Practice Guidelines. Circulation 2019; 139(25): e1082-e143.

78. Hecht H, Blaha MJ, Berman DS, et al. Clinical indications for coronary artery calcium scoring in asymptomatic patients: Expert consensus statement from the Society of Cardiovascular Computed Tomography. J Cardiovasc Comput Tomogr 2017; 11(2): 157-68.

79. Baker CJ, Martinez-Huenchullan SF, D'Souza M, et al. Effect of exercise on hepatic steatosis: Are benefits seen without dietary intervention? A systematic review and meta-analysis. J Diabetes 2021; 13(1): 63-77. 
80. Katsagoni CN, Georgoulis M, Papatheodoridis GV, Panagiotakos DB, Kontogianni MD. Effects of lifestyle interventions on clinical characteristics of patients with non-alcoholic fatty liver disease: A meta-analysis. Metabolism 2017; 68: 119-32.

81. Romero-Gomez M, Zelber-Sagi S, Trenell M. Treatment of NAFLD with diet, physical activity and exercise. J Hepatol 2017; 67(4): 829-46.

82. Lean ME, Leslie WS, Barnes AC, et al. Primary care-led weight management for remission of type 2 diabetes (DiRECT): an open-label, cluster-randomised trial. Lancet 2018; 391(10120): 541-51.

83. Lean MEJ, Leslie WS, Barnes AC, et al. Durability of a primary care-led weight-management intervention for remission of type 2 diabetes: 2-year results of the DiRECT open-label, clusterrandomised trial. The lancet Diabetes \& endocrinology 2019; 7(5): 344-55.

84. Al-Mrabeh A, Hollingsworth KG, Shaw JAM, et al. 2-year remission of type 2 diabetes and pancreas morphology: a post-hoc analysis of the DiRECT open-label, cluster-randomised trial. The lancet Diabetes \& endocrinology 2020.

85. EASL-EASD-EASO Clinical Practice Guidelines for the management of non-alcoholic fatty liver disease. Diabetologia 2016; 59(6): 1121-40.

86. Chalasani N, Younossi Z, Lavine JE, et al. The diagnosis and management of nonalcoholic fatty liver disease: Practice guidance from the American Association for the Study of Liver Diseases. Hepatology 2018; 67(1): 328-57.

87. Glen J, Floros L, Day C, Pryke R. Non-alcoholic fatty liver disease (NAFLD): summary of NICE guidance. Bmj 2016; 354: i4428.

88. Arnett DK, Blumenthal RS, Albert MA, et al. 2019 ACC/AHA Guideline on the Primary Prevention of Cardiovascular\&\#xa0;Disease. Journal of the American College of Cardiology 2019; 74(10): e177-e232. 
89. DeFronzo RA, Inzucchi S, Abdul-Ghani M, Nissen SE. Pioglitazone: The forgotten, costeffective cardioprotective drug for type 2 diabetes. Diabetes \& vascular disease research 2019; 16(2): $133-43$.

90. Musso G, Cassader M, Paschetta E, Gambino R. Thiazolidinediones and Advanced Liver Fibrosis in Nonalcoholic Steatohepatitis: A Meta-analysis. JAMA internal medicine 2017; 177(5): 633-40.

91. Cusi K, Orsak B, Bril F, et al. Long-Term Pioglitazone Treatment for Patients With Nonalcoholic Steatohepatitis and Prediabetes or Type 2 Diabetes Mellitus: A Randomized Trial. Ann Intern Med 2016; 165(5): 305-15.

92. Armstrong MJ, Gaunt P, Aithal GP, et al. Liraglutide safety and efficacy in patients with nonalcoholic steatohepatitis (LEAN): a multicentre, double-blind, randomised, placebo-controlled phase 2 study. Lancet 2016; 387(10019): 679-90.

93. Newsome PN, Buchholtz K, Cusi K, et al. A Placebo-Controlled Trial of Subcutaneous Semaglutide in Nonalcoholic Steatohepatitis. N Engl J Med 2020.

94. Oral Abstracts. Hepatology 2020; 72(S1): 1A-130A. 
Table 1. Meta-analytic quantification of the excess of fatal and nonfatal CVD events, arrhythmias (permanent atrial fibrillation), type 2 diabetes, chronic kidney disease and colorectal tumours in adult individuals with NAFLD.

\begin{tabular}{|c|c|c|c|}
\hline Author(s), Ref. & $\begin{array}{l}\text { Study } \\
\text { characteristics }\end{array}$ & $\begin{array}{l}\text { Study } \\
\text { outcomes }\end{array}$ & $\begin{array}{l}\text { Random-effects } \\
\text { odds ratio or hazard } \\
\text { ratio ( } 95 \% \\
\text { confidence } \\
\text { intervals) }\end{array}$ \\
\hline \multicolumn{4}{|c|}{ Fatal and nonfatal CVD events $\S$} \\
\hline \multirow[t]{6}{*}{ Targher G et al. $^{20}$} & \multirow{4}{*}{$\begin{array}{l}16 \text { longitudinal studies } \\
\text { involving a total of } \\
34,043 \text { individuals } \\
\text { ( } 36.3 \% \text { with NAFLD); } \\
\text { median follow-up of } 6.9 \\
\text { years }\end{array}$} & $\begin{array}{l}\text { Any fatal or nonfatal } \\
\text { CVD events, } n=16 \\
\text { studies }\end{array}$ & $1.64(1.26-2.13)$ \\
\hline & & $\begin{array}{l}\text { Fatal CVD events } \\
\text { (only), } n=6 \text { studies }\end{array}$ & $1.31(0.87-1.97)$ \\
\hline & & $\begin{array}{l}\text { Fatal and nonfatal CVD } \\
\text { events (combined } \\
\text { endpoint), } n=5 \text { studies }\end{array}$ & $1.63(1.06-2.48)$ \\
\hline & & $\begin{array}{l}\text { Nonfatal CVD events } \\
\text { (only), } n=5 \text { studies }\end{array}$ & $2.52(1.52-4.18)$ \\
\hline & \multirow{2}{*}{$\begin{array}{l}\text { Subgroup analyses in } \\
\text { patients with "more } \\
\text { severe" NAFLD ( } n=6 \\
\text { studies)* }\end{array}$} & $\begin{array}{l}\text { Fatal CVD events } \\
\text { (only), } n=3 \text { studies }\end{array}$ & $3.28(2.26-4.77)$ \\
\hline & & $\begin{array}{l}\text { Fatal and nonfatal CVD } \\
\text { events (combined } \\
\text { endpoint), } n=3 \text { studies }\end{array}$ & $1.94(1.17-3.21)$ \\
\hline \multicolumn{4}{|c|}{ Permanent atrial fibrillation } \\
\hline Cai X et al. ${ }^{27}$ & $\begin{array}{l}6 \text { longitudinal studies } \\
\text { involving a total of } \\
614,673 \text { individuals } \\
\text { ( } 40 \% \text { with NAFLD); } \\
\text { median follow-up of } 10 \\
\text { years }\end{array}$ & $\begin{array}{l}\text { Incident atrial } \\
\text { fibrillation, } n=6 \text { studies }\end{array}$ & $1.19(1.04-1.31)$ \\
\hline \multicolumn{4}{|c|}{ Type 2 diabetes mellitus } \\
\hline \multirow[t]{3}{*}{ Mantovani A et al. ${ }^{29}$} & $\begin{array}{l}33 \text { longitudinal studies } \\
\text { involving a total of } \\
501,022 \text { individuals } \\
\text { (30.8\% with NAFLD); } \\
\text { median follow-up of } 5 \\
\text { years }\end{array}$ & $\begin{array}{l}\text { Incident diabetes, } \mathrm{n}=26 \\
\text { studies }\end{array}$ & $2.19(1.93-2.48)$ \\
\hline & \multirow{2}{*}{$\begin{array}{l}\text { Subgroup analyses in } \\
\text { patients with "more } \\
\text { severe" NAFLD } \\
\text { (defined either by } \\
\text { increasing } \\
\text { ultrasonographic scores } \\
\text { or by increasing liver } \\
\text { fibrosis assessed by } \\
\text { histology or noninvasive } \\
\text { fibrosis scores) }\end{array}$} & $\begin{array}{l}\text { Incident diabetes, } \mathrm{n}=9 \\
\text { studies }\end{array}$ & $2.69(2-08-3.49)$ \\
\hline & & $\begin{array}{l}\text { Incident diabetes, } n=5 \\
\text { studies (NAFLD with } \\
\text { increasing severity of } \\
\text { fibrosis assessed by } \\
\text { histology or fibrosis } \\
\text { scores) }\end{array}$ & $3.42(2.29-5.11)$ \\
\hline \multicolumn{4}{|c|}{ Chronic kidney disease (CKD stage $\geq 3$ ) } \\
\hline \multirow[t]{3}{*}{ Mantovani $\mathrm{A}$ et al. ${ }^{32}$} & $\begin{array}{l}13 \text { longitudinal studies } \\
\text { involving a total of } \\
1,222,032 \text { individuals } \\
(28.1 \% \text { with NAFLD); } \\
\text { median follow-up of } 9.7 \\
\text { years }\end{array}$ & $\begin{array}{l}\text { Incident CKD stage } \geq 3 \text {, } \\
n=10 \text { studies }\end{array}$ & $1.43(1.33-1.54)$ \\
\hline & \multirow{2}{*}{$\begin{array}{l}\text { Subgroup analyses in } \\
\text { patients with "more } \\
\text { severe" NAFLD } \\
\text { (defined either by } \\
\text { increasing } \\
\text { ultrasonographic scores } \\
\text { or by increasing liver }\end{array}$} & $\begin{array}{l}\text { Incident CKD stage } \geq 3 \text {, } \\
\mathrm{n}=4 \text { studies }\end{array}$ & $1.67(1.28-2.17)$ \\
\hline & & $\begin{array}{l}\text { Incident CKD stage } \geq 3 \text {, } \\
n=2 \text { studies (severity of } \\
\text { liver fibrosis assessed } \\
\text { by histology) }\end{array}$ & $2.90(1.62-5.18)$ \\
\hline
\end{tabular}




\begin{tabular}{|c|c|c|c|}
\hline & $\begin{array}{l}\text { fibrosis assessed by } \\
\text { histology or noninvasive } \\
\text { fibrosis scores) }\end{array}$ & & \\
\hline \multicolumn{4}{|l|}{ Colorectal tumours } \\
\hline \multirow[t]{4}{*}{ Mantovani A et al. ${ }^{33}$} & \multirow[t]{4}{*}{$\begin{array}{l}8 \text { cross-sectional and } 3 \\
\text { longitudinal studies } \\
\text { involving a total of } \\
91,124 \text { asymptomatic } \\
\text { individuals undergoing } \\
\text { screening colonoscopy } \\
(32.1 \% \text { with NAFLD) }\end{array}$} & $\begin{array}{l}\text { Prevalent colorectal } \\
\text { adenomas, } n=8 \text { studies }\end{array}$ & $\begin{array}{l}1.28 \text { (1.11-1.48), } \mathrm{n}=7 \\
\text { studies using liver } \\
\text { imaging techniques } \\
\text { for NAFLD diagnosis } \\
1.61(0.90-2.89), \mathrm{n}=1 \\
\text { study using liver } \\
\text { histology for NAFLD } \\
\text { diagnosis }\end{array}$ \\
\hline & & $\begin{array}{l}\text { Prevalent colorectal } \\
\text { cancer, } n=5 \text { studies }\end{array}$ & $\begin{array}{l}1.56 \text { (1.25-1.94), } \mathrm{n}=4 \\
\text { studies using liver } \\
\text { imaging techniques } \\
3.04 \text { (1.29-7.18), } \mathrm{n}=1 \\
\text { study using liver } \\
\text { histology for NAFLD } \\
\text { diagnosis }\end{array}$ \\
\hline & & $\begin{array}{l}\text { Incident colorectal } \\
\text { adenomas, } n=3 \text { studies }\end{array}$ & $\begin{array}{l}1.42(1.18-1.72), n=3 \\
\text { studies using liver } \\
\text { imaging techniques } \\
\text { for NAFLD diagnosis }\end{array}$ \\
\hline & & $\begin{array}{l}\text { Incident colorectal } \\
\text { cancer, } n=1 \text { study }\end{array}$ & $\begin{array}{l}3.08(1.02-9-03) \text { using } \\
\text { liver imaging } \\
\text { techniques for NAFLD } \\
\text { diagnosis }\end{array}$ \\
\hline
\end{tabular}

$\S$ Fatal and/or nonfatal CVD events were defined as presence of cardiovascular death, nonfatal CVD events (i.e., acute myocardial infarction, angina, ischemic stroke or coronary revascularization procedures), or both.

* Defined by the presence of either NAFLD on imaging techniques plus either elevated serum gamma-glutamyltransferase concentrations or high NAFLD fibrosis score or high F-18 fluoro-deoxyglucose uptake on positron emission tomography, or by increasing severity of liver fibrosis on histology. 